\title{
Auto-control of pumping operations in sewerage systems by rule-based fuzzy neural networks
}

\author{
Y.-M. Chiang ${ }^{1}$, L.-C. Chang ${ }^{2}$, M.-J. Tsai ${ }^{1}$, Y.-F. Wang ${ }^{3}$, and F.-J. Chang ${ }^{1}$ \\ ${ }^{1}$ Department of Bioenvironmental Systems Engineering, National Taiwan University, Taipei, Taiwan \\ ${ }^{2}$ Department of Water Resources and Environmental Engineering, Tamkang University, Taipei, Taiwan \\ ${ }^{3}$ Water Resources Agency, Ministry of Economic Affairs, Taiwan
}

Received: 26 July 2010 - Published in Hydrol. Earth Syst. Sci. Discuss.: 6 September 2010

Revised: 7 December 2010 - Accepted: 25 December 2010 - Published: 19 January 2011

\begin{abstract}
Pumping stations play an important role in flood mitigation in metropolitan areas. The existing sewerage systems, however, are facing a great challenge of fast rising peak flow resulting from urbanization and climate change. It is imperative to construct an efficient and accurate operating prediction model for pumping stations to simulate the drainage mechanism for discharging the rainwater in advance. In this study, we propose two rule-based fuzzy neural networks, adaptive neuro-fuzzy inference system (ANFIS) and counterpropagation fuzzy neural network for on-line predicting of the number of open and closed pumps of a pivotal pumping station in Taipei city up to a lead time of $20 \mathrm{~min}$. The performance of ANFIS outperforms that of CFNN in terms of model efficiency, accuracy, and correctness. Furthermore, the results not only show the predictive water levels do contribute to the successfully operating pumping stations but also demonstrate the applicability and reliability of ANFIS in automatically controlling the urban sewerage systems.
\end{abstract}

\section{Introduction}

Taiwan is located in subtropical zone with frequent thunderstorms and typhoon events. Both temporal and spatial distributions of precipitation are uneven due to the mountainous topography which occupies about $70 \%$ of Taiwan. In addition, the time of concentration in a metropolitan area is reduced and both the rate and amount of surface runoff increase owing to more impervious areas caused by urbanization. As

Correspondence to: F.-J. Chang

(changfj@ntu.edu.tw) a result, the phenomenon of flood/flooding in urbanized area will occur if the surface runoff exceeds the design capacity of the sewerage system. For example, Typhoon Nari brought massive rainfalls at an astonishing level of $500 \mathrm{~mm} /$ day on 17 September 2001 which resulted in 27 deaths and countless economic losses.

In case of flood/flooding in urban areas, the operation strategy for sewerage systems in Taipei City is to set up pumping stations which are the major hydraulic facilities for inner rainwater discharges. Undoubtedly, pumping stations play a key role in flood reduction in metropolitan areas. Nevertheless, fast rising peak flows resulting from urbanization and climate change are highly challenging to existing sewerage systems. In fact, the current pumping operation procedure depends more highly on the experiences of local operators than on the pumping operation standards. In other words, there are no explicit guidelines for pumping operations. Operators have to stand by prior to the coming of extreme rainfall events and keep monitoring and operating until storms' departure. It is time- and human resourcesconsuming with no guarantee of safe pumping operations because only the information of current water level measurements is available for operators. Therefore, it is necessary to construct an efficient and accurate pumping operation model to simulate the drainage mechanism for discharging rainwater in advance. Furthermore, the advantages of building a suitable and successful pumping operation prediction model for a sewerage system are to increase its storage capacity prior to peak flows by reducing water levels in advance and to decrease flood/flooding probability by speeding up discharge rates during storm periods. To achieve this goal, two rulebased fuzzy neural networks are introduced in this study by taking the predictive water levels into account to effectively

Published by Copernicus Publications on behalf of the European Geosciences Union. 
on-line predict the number of open pumps for a pivotal pumping station in Taipei city during the process of storm events.

The combination of an artificial neural network (ANN) and a fuzzy theory into a fuzzy neural network has proven to be another powerful intelligent system and has received much attention in recent years (Chang et al., 2005; Coulibaly and Evora, 2007; Firat, 2008; Nayak et al., 2004; Wang et al., 2009; Yarar et al., 2009). There are several reasons for adopting fuzzy neural networks as effective operating models. In general, fuzzy neural networks require less information than physical/stochastic models. Besides, physi$\mathrm{cal} / \mathrm{stochastic}$ models are usually more complex, relying on the skill and experience of the modeler, whereas the advantage of the fuzzy neural networks is that it does not require the model structure to be known a priori. Furthermore, the individual strengths of ANN and fuzzy logic approaches can be exploited in a synergistic way for the hybrid construction of fuzzy neural network systems. Therefore, the utilization of fuzzy neural networks is an attractive approach especially when dealing with control systems. For example, Chang and Chang (2001) proposed a fuzzy neural network for reservoir operation and showed that the network improves the efficiency of operation than the classical models based on rule curves.

Both ANNs and fuzzy theories are state-of-the-art technologies that try to mimic the human thinking process for learning similar strategies or experiences to make optimal decisions, and are well recognized for their outstanding abilities in modeling complex nonlinear systems such as precipitation estimation/prediction (Chiang et al., 2007), streamflow forecasting (Abrahart and See, 2002; Brath et al., 2002; Chiang et al., 2004; Dawson et al., 2002; Shrestha and Nestmann, 2009; Toth, 2009), reservoir operations (Chaves and Kojiri, 2007; Hsu and Wei, 2007; Mehta and Jain, 2009; Pinthong et al., 2009), prediction of water quality parameters (Sudheer et al., 2006; Tyagi et al., 2008), and pumping operations (Chang et al., 2008; Rao et al., 2007).

ANNs are effective in extracting significant features from complex databases and are capable of learning the relationship between any data pairs. Fuzzy theories are based on the way how brains deal with inexact information. The fundamental inspirations of these two methods are quite different. ANNs offer good performance in dealing with sensory data, while fuzzy systems often deal with issues such as reasoning at a higher analytical level than ANNs. However, due to the lesser learning capability of fuzzy systems, it is difficult to tune the fuzzy rules and membership functions from training datasets. Thus, a promising approach to reaping and capturing the strengths and benefits of a fuzzy system and an artificial neural network is to merge them into a hybrid system with a single framework. In this study, we evaluated the performance of two rule-based fuzzy neural networks fed with different input combinations for predicting pumping operations up to a forecast lead-time of $20 \mathrm{~min}$. Furthermore, methodologies and a brief description of two rule-based fuzzy neural networks were presented. In the application procedure, a study area and data set were first given, and the fuzzy neural network-based pumping operation models were then performed. Next, the results of two fuzzy neural networks were separately discussed. Finally, a conclusion of this study was drawn.

\section{Methodologies}

We applied two popular rule-based fuzzy neural networks, namely counterpropagation fuzzy neural network (CFNN) and adaptive neuro-fuzzy inference system (ANFIS), for establishing the proper relationship between hydrological factors and pumping operations in an urban sewerage system.

\subsection{Counterpropagation Fuzzy Neural Network (CFNN)}

The counterpropagation network (CPN), which was proposed by Hecht-Nielsen (1987), functions as a selfprogramming optimal lookup table, providing the mapping between input and output patterns. CFNN, a combination of CPN and fuzzy arithmetic, was first introduced by Nie and Linkens (1994). The architecture of CFNN consists of an input layer, a Kohonen layer, and a Grossberg layer (see Fig. 1). Each neuron arranged in the Kohonen layer represents a rule. The connections between input and Kohonen layers indicate the "if" statement of a rule. The connections between Kohonen and Grossberg layers represent the "then" part of a rule. CFNN has a hybrid learning scheme and can be split into two stages during model construction. In the first stage, the model with unsupervised learning is used to build the if-then rule according to the similar characteristics of input vectors; while the weight vectors between the Kohonen and Grossberg layers are adjusted by using supervised learning to improve the performance in the second stage. CFNN is also a good pattern recognition engine and a robust classifier that has the capability of making decisions based on imprecise input data. Chang et al. (2008) indicated the network has a simple basic structure with efficient learning ability to construct a human-like operating strategy for operating the flood control system. The detailed procedure for constructing CFNN can be found in Chang and Chen (2001) and is briefly described as follows.

\subsubsection{Learning procedure of the CFNN}

An input vector $X=\left(x_{1}, \ldots, x_{i}, \ldots, x_{n}\right)$ would be transformed to an output vector $Y=\left(y_{1}, \ldots, y_{m}\right)$ through a logical judgment, which is called a set of rules. The connections between the input layer and the Kohonen layer are indicated as $w$ which is an "if" statement of rule-base control, while the connections between the Kohonen layer and the Grossberg layer are $\pi$ which is the "then" statement of rule-base control. Thus, the statement of each rule is defined as: "if $X$ is $w$, 


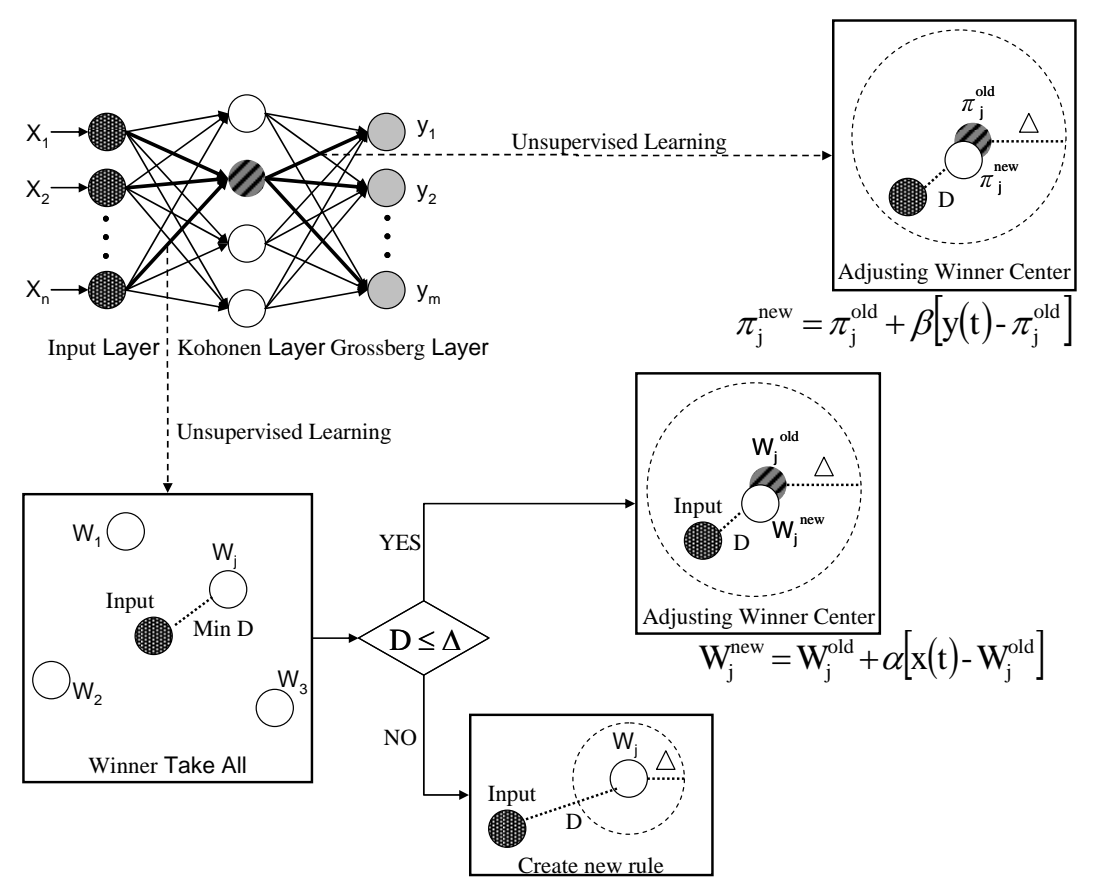

Fig. 1. The architecture of a CFNN and its training procedure.

then $Y$ is $\pi$ ". Weights arranged in the Kohonen and Grossberg layers are, respectively, trained by the following steps. In the Kohonen layer, each neuron represents a rule. First, a $\Delta$ value should be selected before the learning processes. The meaning of $\Delta$ represents the width of the rule. Data with distance (input data and the nearest center) less than $\Delta$ are classified into the same rule. In this study, the value of $\Delta$ is optimized by using trial-and-error method. Second, the distances $\left(D_{j}\right)$ between each input $X(t)$ and the centers $w_{j}$ of the existing neurons are calculated. The neuron with minimum distance is regarded as the winning neuron. If the minimum distance is smaller than $\Delta$, the center of $w$ and $\pi$ has to be updated as

$w_{j}^{\text {new }}=w_{j}^{\text {old }}+\alpha\left[X(t)-w_{j}^{\text {old }}\right]$

$\pi_{j}^{\text {new }}=\pi_{j}^{\text {old }}+\beta\left[Y(t)-\pi_{j}^{\text {old }}\right]$

where $\alpha$ and $\beta$ are learning rates within the interval $[0,1]$ and calibrated during the model training phase. $Y(t)$ is the output vector. If the minimum distance is larger than $\Delta$, a new rule will be created as $w_{j}^{\text {new }}=X(t), \pi_{j}^{\text {new }}=Y(t)$, and the existing $N$ rules will become $(N+1)$ rules. Figure 1 also shows the training procedure of a CFNN. Generally speaking, the number of rules and the model accuracy will gradually increase when the value of $\Delta$ increases. However, the complexity of the network is also proportional to the value of $\Delta$.

\subsubsection{Forecasting procedure of the CFNN}

The forecasting procedure of CFNN consists of pattern matching and weighted average, and is coupled with the concept of fuzzy control. Pattern matching uses the Gaussian membership function $\left(s_{j} \in[0,1]\right)$ for calculating the distance between the input and the $j$ th rule to assign a degree of membership to each rule. The use of Gaussian function has been frequently suggested in previous works and popularly applied for various fields. Besides, based on our previous experiences, the Gaussian function generally provided more flexible degree of membership and thus produced more appropriate results than the triangular function (Chang and Chang, 2001). Therefore, the Gaussian function was selected to represent the degree of membership. If the distance is larger than $\Delta$, then $s_{j}=0$. It means the impact of the $j$ th rule on the input is null. The fuzzy control output $\hat{Y}(t)$ is then the weighted average of the output of each rule and can be calculated as follows.

$\hat{Y}(t)=\frac{\sum_{j=1}^{N} s_{j} \pi_{j}}{\sum_{j=1}^{N} s_{j}}$

\subsection{Adaptive Neuro-Fuzzy Inference System (ANFIS)}

Adaptive Neuro-Fuzzy Inference System (ANFIS) proposed by Jang (1993) is a multi-layer feed-forward neural network that combines ANN and fuzzy logic. It eliminates the basic problem in fuzzy system design, which defines the membership functions and designs fuzzy rules, by effectively 
using the learning capability of ANN for automatic fuzzy rule generation and parameter optimization (Nayak et al., 2004). Moreover, ANFIS not only maintains the mapping ability of ANNs but also possesses the advantages of fuzzy if-then rules for describing the local behavior of such mapping and solving the highly non-linear control problem robustly. ANFIS has been widely studied and successfully applied to hydrology and water resources, such as Chang and Chang (2006).

Generally, the architecture of ANFIS consists of five layers. The first layer consists of input nodes where each node corresponds to a linguistic label with a membership function (MF). In this study, a bell-shaped MF is used. The output of the first layer specifies the degree where the given input satisfies the MF. The second layer consists of rule nodes and the output of each node represents the firing strength of a rule. The node generates its output by multiplying all incoming signals involved in the rule. Therefore, the outputs of this layer are the products of the corresponding degrees from Layer 1. The third layer consists of average nodes that compute the ratio of each rule's firing strength to the sum of all rules' firing strength. The fourth layer consists of consequent nodes. The function of consequent nodes is to compute the contribution of each rule towards the total output. The fifth layer consists of output nodes. This layer includes a stable single node that sums up values of all signals to calculate the final output.

The training of ANFIS is based on a hybrid supervised learning algorithm which is a combination of a gradient descent method and a least-squares method. These algorithms are employed to optimize both linear and nonlinear parameters. Furthermore, it is very important to define the fuzzy rules when designing an ANFIS model because the number of determined parameters may increase enormously as the number of rules increases. A solution to this problem is to use the subtractive fuzzy clustering algorithm (SFCA) to establish the rule-based relationship between input and output variables. The SFCA is devoted to the automatic determination of the minimum number of rules to discriminate the fuzzy quality associated with each cluster. The algorithm calculates the measure of likelihood of each data, which defines the cluster center based on the density of surrounding data points. Details of the ANFIS algorithm coupled with the SFCA can be found Chang and Chang (2001).

\section{Applications}

\subsection{Study area and data}

Taipei City is situated in the Taipei Basin where the Danshuei River wanders through the area. The average elevation of the area is only four meters above sea level. The topography of Danshuei estuary is also narrow and therefore the ground runoffs concentrated from the city cannot be effectively dis- charged by gravity during typhoon periods. Storm water in Taipei is drained by pumping stations which are the principal hydraulic facilities for flood discharges, and therefore play an important role in flood mitigation. The site under consideration is the Yu-Cheng catchment, located in southeastern Taipei as shown in Fig. 2, which is chosen for a detailed investigation of different input strategies on pumping operations. The catchment with an area of about 1645 ha has the biggest sewerage system in Taipei City. This region has five rain gauging stations denoted by blue circles in Fig. 2. The outlet of the sewerage system is the water level gauging station, YC10, marked by a red triangle. The Yu-Cheng pumping station, marked by a purple square, was built in 1987 for the purpose of pumping the inner water into the Keelung River. This pumping station contains seven massive pumps (see Fig. 2) with a total capacity of $184.1 \mathrm{~m}^{3} / \mathrm{s}$ and was the most advanced and largest one in Asia in the 1980s. In general, the gravity gates of Yu-Cheng pumping station remain open to drain away the inner water to the Keelung River. When the water level of Keelung River rises up to the warning level $(1.8 \mathrm{~m})$, the pumps will be turned on and warmed up. These pumps will start pumping when the water level of Keelung River is higher than $2.4 \mathrm{~m}$; meanwhile, the gravity gates are immediately closed, which means running water cannot be discharged by gravity. These seven pump sets are operated sequentially according to the changes of water levels, which means when a running pump cannot control the water level under the level of $2.4 \mathrm{~m}$, another pump will start working together with the first one.

Due to global climate change and urbanization, extreme rainfall events usually bring torrential rainwater and result in fast flood in Taiwan. The original design of the operating procedure is not able to accommodate the rainfall-runoff phenomenon nowadays. As current operating mechanism, there are no explicit guidelines for pumping operations but are highly dependent on the experience of operators. In sum, the pumping station is operated under actual circumstances and requires experienced operators to adjust the status of the pumps. To overcome the disadvantages of current operations, it is necessary to construct an efficient and accurate pumping operation model to simulate the drainage mechanism for discharging rainwater and reducing the risk of flood in advance.

Because YC10 is the outlet of the sewerage system, there is a high correlation between water levels and the operational strategies of the Yu-Cheng pumping station. Therefore, water level measurements at YC10 were collected from 2002 to 2008 and consisted of 17 storm events. This information was further calculated to obtain the lifts of different duration $(\Delta L(t)$ : the difference of water levels between time $t$ and $t-5)$. Meanwhile, the precipitation, status of four gravity gates (open or close), and records of seven pumps at the same observational periods were also collected. The precipitation observation values collected at five rain gauges were used to calculate the mean areal rainfall $(R(t))$ based on the Thiessen polygon method in order to effectively reduce the 


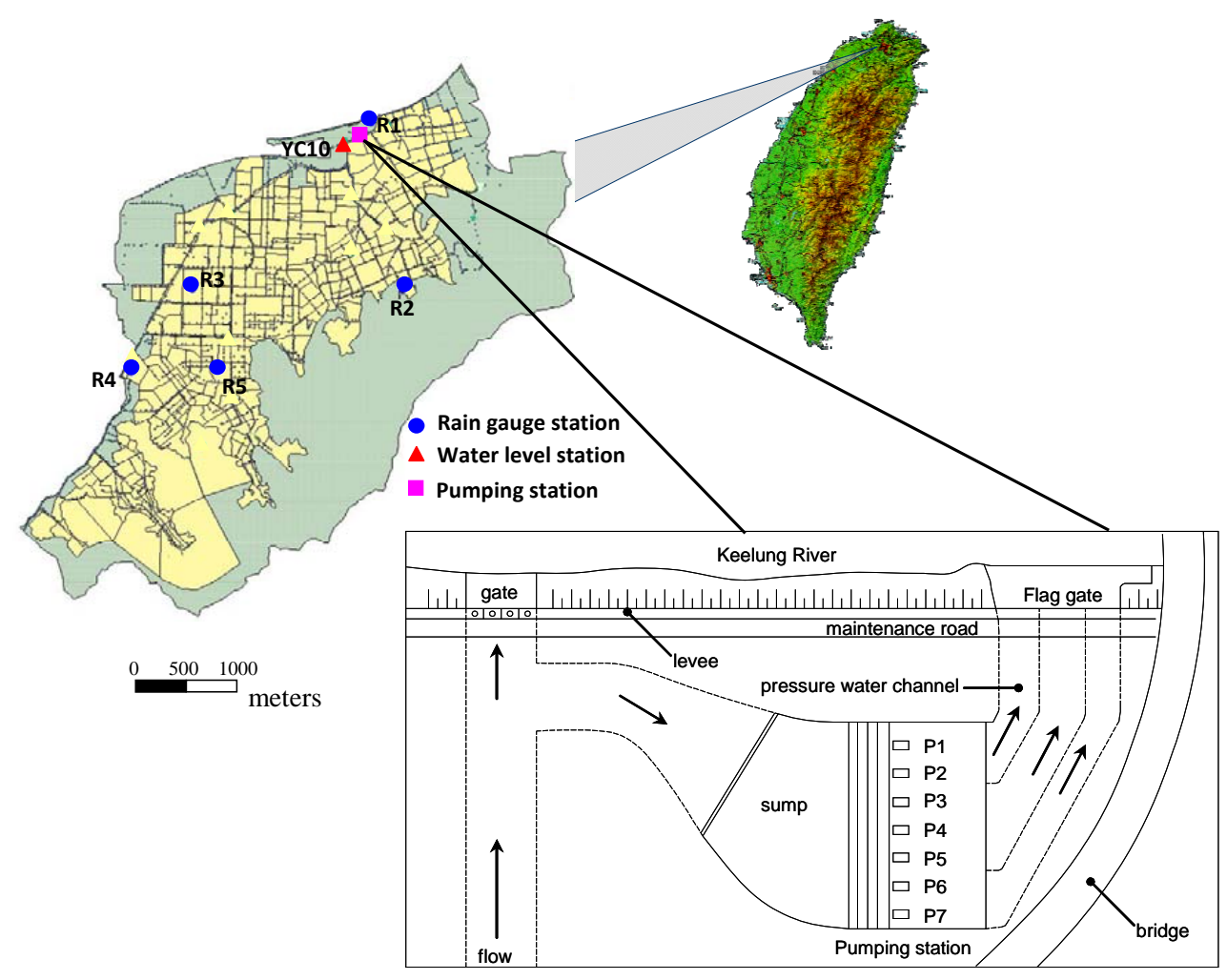

Fig. 2. Location of the study area and gauging stations and the design of Yu-Cheng pumping station.

input dimension of the fuzzy neural networks. Regarding the information on gravity gates, the value is recorded as 1 when the gate is open; whereas the value is recorded as 0 when the gate is closed. The status of four gravity gates is then summed up as one input variable $(G(t))$. As far as the operation of pumps is concerned, the recording rule is the same as that of gravity gates ( 1 means open; 0 means closed). Accordingly, the status of seven pumps is then summed up as one input variable $(P(t))$. The activation of 7 pumps is operated in a pre-designed order, which makes no difference of the discharge capability for individual pumps. Prior information of the total number of running pumps can be a great reference to operators, especially when a fast flood occurs. By investigating the pumping operation, the total number of running pumps for model output is a simple and meaningful variable and directly fits the need of operators.

Besides, another important piece of information, the water level predictions $\left(L^{\prime}(t+5), L^{\prime}(t+10), L^{\prime}(t+15)\right.$, $\left.L^{\prime}(t+20)\right)$ at YC10 performed in our previous study (Chiang et al., 2010) were also conducted in this study as an additional input. The predictive water levels were obtained from a three-layer recurrent neural network (RNN) with internal time-delay feedback loops in both hidden and output layers. For predicting the water level of Station YC10, the input information to the RNN-based hydrological model mostly came from the mean areal precipitations and two upstream water level gauging stations that belong to two sub-drainage systems. The learning target of the RNN model was the water level observations of YC10. To obtain 5- to 20-min-ahead water level predictions, this study constructed four identical RNN structures, each with a single output. The calibration of model parameters was performed by the gradient descent method via minimizing the forecasting errors. The results show that the RNN is capable of producing satisfactory predictions for 5-, 10-, 15-, and 20-min-ahead water level predictions at YC10. The coefficient of efficiency (CE) between the predictive water levels and observations remained 0.95 for 20-min-ahead prediction in testing phase (see Table 1), indicating that the accuracy of water level predictions was high and should be helpful for modeling pumping operations.

After data preprocessing, these data were normalized into the interval $[0,1]$ before they were input into the models. A total of 2375 records of data, extracted from 17 typhoon or storm events, with a temporal resolution of $5 \mathrm{~min}$ were collected. These data were divided into three different phases: training, validation, and testing. Data associated with eight events were arranged in a training phase for model learning purposes, data associated with the other three events were dedicated to validate the optimal neural networks, and the remaining six events were used for testing the generation capability of rule-based fuzzy neural networks. 
Table 1. Results of water level prediction at YC10 in terms of CE.

\begin{tabular}{lrrrr}
\hline & \multicolumn{4}{c}{ Lead time } \\
\cline { 2 - 5 } & 5 min. & 10 min. & 15 min. & 20 min. \\
\hline Training & 0.99 & 0.99 & 0.98 & 0.97 \\
Validation & 0.99 & 0.98 & 0.95 & 0.93 \\
Testing & 0.99 & 0.99 & 0.97 & 0.95 \\
\hline
\end{tabular}

\subsection{Fuzzy neural network-based pumping operation models}

There is no other traditional approach being used to simulate the pumping system. One way to show how a system responds to an input is to collect the historical records and then analyze the input-output relation. If a robust relation is found, it can be used for future operation when similar input situation occurs. Therefore, we proposed the rule-based fuzzy neural networks that learned from circumstances, predictive information, and historical pumping operations made by the experienced operators. This is the very first study using the AI techniques to simulate the drainage mechanism for discharging rainwater and reducing the risk of flood in advance.

In this study, two rule-based fuzzy neural networks were built for predicting the operation of pumps in Yu-Cheng pumping station by taking observational and forecasting information into account (see Fig. 3). Thus, two types of input strategies (conventional and improved types) were designed for CFNN and ANFIS pumping operation models, separately. The conventional type only consists of observation data, whereas the improved type not only considers the measurements but also adopts the water level predictions. The main purpose is to investigate the impact of predictive water levels on pumping operation predictions. Therefore, the conventional type has four identical structures, each with a single output, designed for 5-, 10-, 15-, and 20-min-ahead predictions of pumping operations. The time step is set as 5 min because the operational time step of the Yu-Cheng pumping station is $5 \mathrm{~min}$. Table 2 shows the input-output combinations for both CFNN and ANFIS models. It should be noted that the improved type has four different cases with different predictive information. Case 1 is only fed with additional information of 5-min-ahead water level predictions, Case 2 is fed with information of 5- and 10-min-ahead water level predictions, Case 3 is fed with information of 5-, $10-$, and 15-min-ahead water level predictions, and Case 4 is fed with information of 5-, 10-, 15-, and 20-min-ahead water level predictions. Accordingly, each input case was also used for constructing pumping operation prediction models up to 20 min-ahead. As the input information changes, the number of output reduces. For example, Case 4 only produces pumping operation predictions at 20-min-ahead because the input information involves 20-min-ahead water level prediction. In sum, the improved type consisted of ten models fed with different input combinations and were designed for both CFNN and ANFIS.

As far as rule-based neural networks are concerned, it is important to determine the appropriate number of rules. Having too many rules may result in similarities between two or more rules, and therefore consume a great deal of computation time; whereas, too few rules may not supply the network with sufficient capability to effectively describe the relation between inputs and outputs. Therefore, the design of $\Delta$ for CFNN and radius ( $r$ ) for ANFIS is a crucial step to enable the models to have better generalization ability. Because there is no specific method suggested for the determination of these parameters, a trial-and-error procedure is adopted in this study. The initial setting of $\Delta$ is set as 0.05 and is increased 0.01 in each step until $\Delta$ reaches 0.8 . Besides, the initial values of learning rates $\alpha$ and $\beta$ are set to 0.5 and the limit of fault tolerance is 0.0001 for CFNN. As for the ANFIS model, the initial setting of the radius is set as 0.1 and is increased 0.01 in each step until the value of radius reaches 0.9. The mean square error (MSE) is taken as a criterion to determine these parameters via judging the error between model outputs and actual operating values. Both MSE and mean absolute error (MAE) are used for the evaluation of model performance. In general, MSE is used as a common assessment index and usually results in larger errors that occur in the vicinity of high values; whereas MAE computes all deviations from the original data series and is not weighted towards high values. Both of these indices are widely used to estimate the fitness to the hydrological models, and therefore to facilitate the comparison of different predictive results. These criteria are defined as follows.

$\mathrm{MSE}=\frac{\sum_{i=1}^{N}\left(P^{\prime}(t)-P(t)\right)^{2}}{N}$

MAE $=\frac{\sum_{i=1}^{N}\left|\left(P^{\prime}(t)-P(t)\right)\right|}{N}$

where $P^{\prime}(t)$ and $P(t)$ are the numbers of active pumps obtained from model outputs and observational records, respectively.

\section{Results and discussion}

\subsection{Results obtained from CFNN}

Table 3 shows the results of pumping operations obtained from CFNN for both conventional and improved types. First, the performance is obviously reduced with the increase of forecasted lead time, no matter which type it is. Besides, the optimal number of rules for these CFNN-based models is 
Table 2. The input and output patterns of CFNN and ANFIS models for predictions.

\begin{tabular}{|c|c|c|c|}
\hline Types & Case & Input & Output \\
\hline \multicolumn{2}{|c|}{ Conventional } & $\Delta L(t), \Delta L(t-5), R(t), R(t-5), G(t), P(t)$ & $\begin{array}{l}P^{\prime}(t+5) \\
P^{\prime}(t+10) \\
P^{\prime}(t+15) \\
P^{\prime}(t+20)\end{array}$ \\
\hline \multirow{4}{*}{ Improved } & 1 & $\Delta L^{\prime}(t+5), \Delta L(t), R(t), R(t-5), G(t), P(t)$ & $\begin{array}{l}P^{\prime}(t+5) \\
P^{\prime}(t+10) \\
P^{\prime}(t+15) \\
P^{\prime}(t+20)\end{array}$ \\
\hline & 2 & $\Delta L^{\prime}(t+10), \Delta L^{\prime}(t+5), \Delta L(t), R(t), R(t-5), G(t), P(t)$ & $\begin{array}{l}P^{\prime}(t+10) \\
P^{\prime}(t+15) \\
P^{\prime}(t+20)\end{array}$ \\
\hline & 3 & $\Delta L^{\prime}(t+15), \Delta L^{\prime}(t+10), \Delta L^{\prime}(t+5), \Delta L(t), R(t), R(t-5), G(t), P(t)$ & $\begin{array}{l}P^{\prime}(t+15) \\
P^{\prime}(t+20)\end{array}$ \\
\hline & 4 & $\begin{array}{l}\Delta L^{\prime}(t+20), \Delta L^{\prime}(t+15), \Delta L^{\prime}(t+10), \Delta L^{\prime}(t+5), \Delta L(t), R(t), \\
R(t-5), G(t), P(t)\end{array}$ & $P^{\prime}(t+20)$ \\
\hline
\end{tabular}

Remark: all $\Delta L^{\prime}(t+n)$ was calculated from $L^{\prime}(t+n)-L^{\prime}(t+n-5) n=5,10,15$, and 20 .

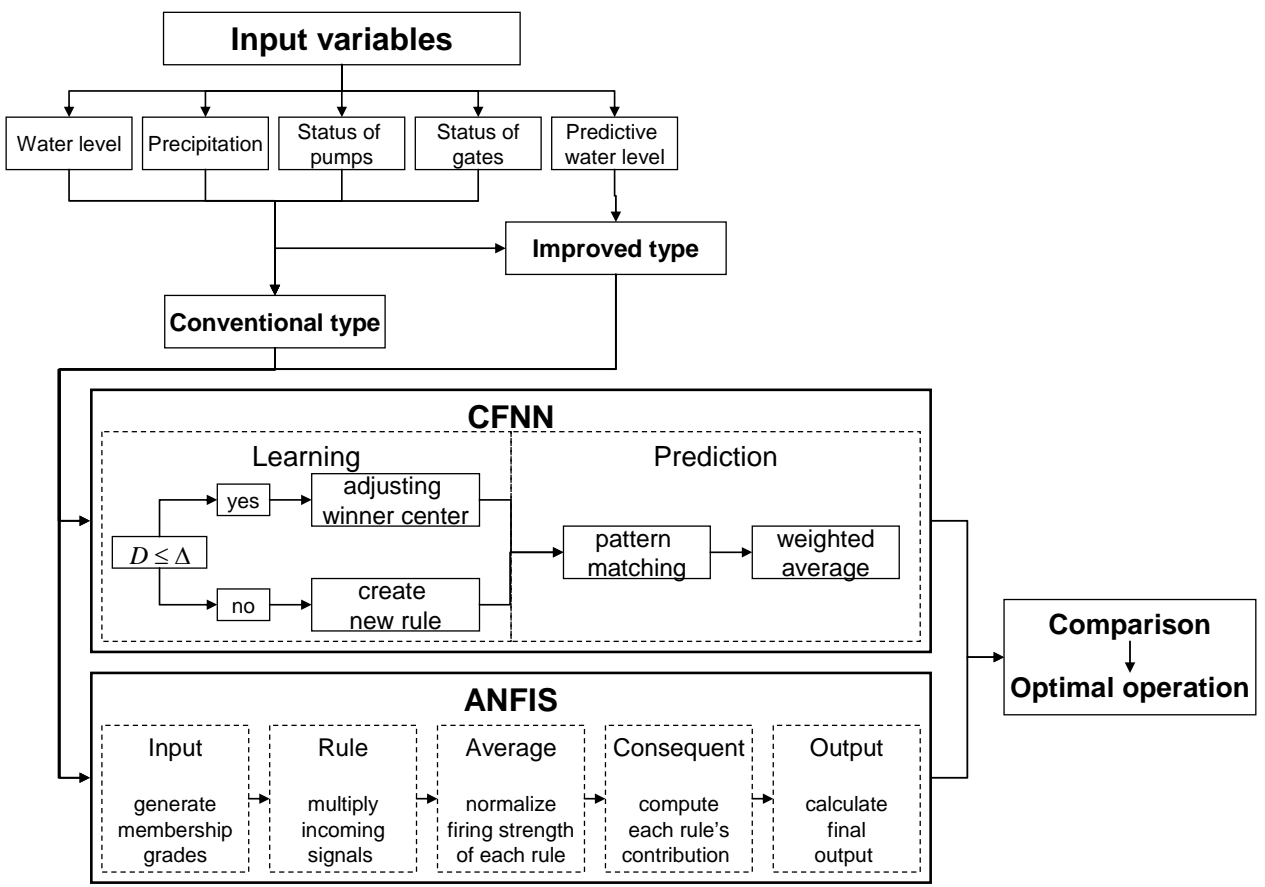

Fig. 3. The flowchart of model construction.

within the interval of $[15,28]$ which is related to the value of $\Delta$ and the complexity of input-output patterns. In other words, the very complex data need smaller $\Delta$ to derive a suitable number of rules, and a smaller $\Delta$ usually results in more rules. In our previous work (Chang et al., 2008), the CFNN was investigated to predict the pumping operation for 5-min-ahead by being fed with observational data which has a similar concept to conventional type $(t+5)$., In this study, the CFNN was further extended for predicting pumping operation up to a lead time of $20 \mathrm{~min}$. Besides, the predictive water level was expected to provide useful information and thus was conducted as an additional model input herein. It is 
Table 3. Results obtained from CFNN for conventional and improved types.

\begin{tabular}{|c|c|c|c|c|c|c|}
\hline \multirow[b]{2}{*}{ Lead time } & \multicolumn{3}{|c|}{ MAE } & \multicolumn{3}{|c|}{ MSE } \\
\hline & training & validation & testing & training & validation & testing \\
\hline & \multicolumn{6}{|c|}{ Conventional type } \\
\hline$t+5$ & 0.59 & 0.18 & 0.11 & 1.18 & 0.22 & 0.16 \\
\hline$t+10$ & 1.16 & 0.93 & 0.94 & 1.88 & 1.25 & 1.04 \\
\hline$t+15$ & 1.94 & 1.64 & 2.26 & 4.82 & 3.39 & 5.69 \\
\hline \multirow[t]{2}{*}{$t+20$} & 1.96 & 1.64 & 2.28 & 4.88 & 3.49 & 5.77 \\
\hline & \multicolumn{6}{|c|}{ Improved type-Case 1} \\
\hline$t+5$ & 0.27 & 0.03 & 0.05 & 0.45 & 0.04 & 0.06 \\
\hline$t+10$ & 0.36 & 0.08 & 0.06 & 0.59 & 0.10 & 0.08 \\
\hline$t+15$ & 0.49 & 0.12 & 0.14 & 0.98 & 0.16 & 0.19 \\
\hline \multirow[t]{2}{*}{$t+20$} & 0.59 & 0.16 & 0.16 & 1.12 & 0.23 & 0.25 \\
\hline & \multicolumn{6}{|c|}{ Improved type-Case 2} \\
\hline$t+10$ & 0.27 & 0.11 & 0.04 & 0.47 & 0.14 & 0.06 \\
\hline$t+15$ & 0.46 & 0.18 & 0.10 & 0.69 & 0.22 & 0.13 \\
\hline \multirow[t]{2}{*}{$t+20$} & 0.48 & 0.30 & 0.16 & 0.77 & 0.43 & 0.24 \\
\hline & \multicolumn{6}{|c|}{ Improved type-Case 3} \\
\hline$t+15$ & 0.43 & 0.10 & 0.09 & 0.94 & 0.14 & 0.13 \\
\hline \multirow[t]{2}{*}{$t+20$} & 0.46 & 0.29 & 0.16 & 0.76 & 0.42 & 0.24 \\
\hline & \multicolumn{6}{|c|}{ Improved type-Case 4} \\
\hline$t+20$ & 0.39 & 0.26 & 0.15 & 0.67 & 0.36 & 0.20 \\
\hline
\end{tabular}

Note: the gray shaded cells indicate the best results at each forecasted lead time.

clear that all of the ten structures from four improved cases performed better than those of the conventional type, no matter what the forecast lead time was (see Table 3), which indicates that the information from water level predictions do contribute to the predictions of pumping operations and effectively improve the accuracy of model outputs as compared with the conventional type.

As far as the same forecast lead time is concerned, another important result can be found among different improved cases. The finding is that the longer the lead time is, the better the performance is produced by the model fed with the latest predictive water level. For example, for predictions at a lead time of $20 \mathrm{~min}$ ahead by CFNN, Case 4 not only produces better results than conventional types but also outperforms other improved cases. This is mainly because the structure of Case 4 includes information of future water levels up to $20 \mathrm{~min}$. The same scenarios can be found for model predictions at other lead times. Consequently, the best results for lead times of 5, 10, 15, and $20 \mathrm{~min}$ are derived from improved Case 1, Case 2, Case 3, and Case 4, respectively, (refer to the rows marked in gray in Table 3). Figure 4 illustrates the best testing result at each forecast lead time. The blue line indicates the measured water level, while the black dashed line and the red dotted line represent the actual records of running pumps and the number of running pumps predicted by models, respectively. As expected, the CFNN-based pumping operation models provide accurate predictions with slight underestimation in the vicinity of peak values for lead times of 5 and $10 \mathrm{~min}$. As for 15 min-ahead predictions, the phenomenon of underestimation increased in the period of 700-900 min, whereas the model overestimated the numbers of running pumps from 900 to $1300 \mathrm{~min}$ for 20 -min-ahead predictions.

\subsection{Results obtained from ANFIS and comparison}

Tables 4 and 5 show the results obtained from ANFIS for conventional and improved types in validation and testing phases, respectively. The results of different models accomplished in validation phase are corresponding with those of testing phase, indicating that the ANFIS models were well trained and had no over-fitting problems. Basically, the performances of different types of ANFIS-based pumping operation models are similar to those of CFNN-based models. That is, all models with improved structures produce better predictions than those with conventional structures. And the best results achieved by ANFIS models for each forecast lead time are identical to those of CFNN models. As the gray 


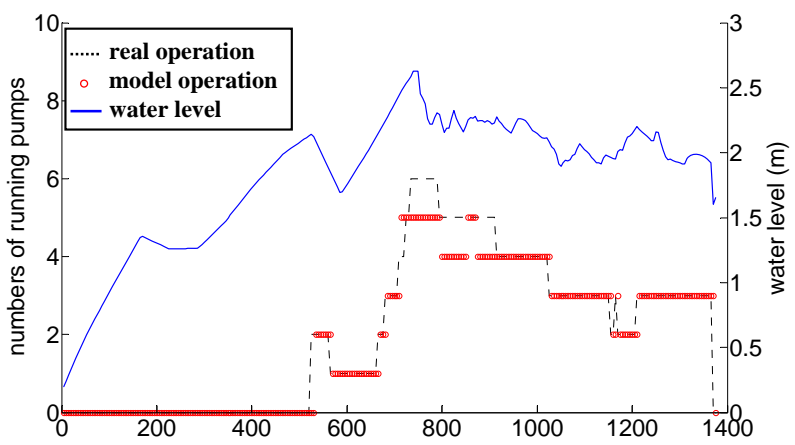

(a)

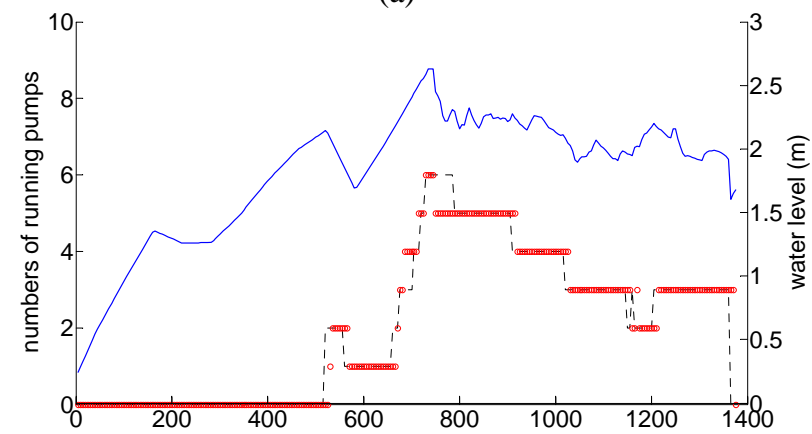

(b)

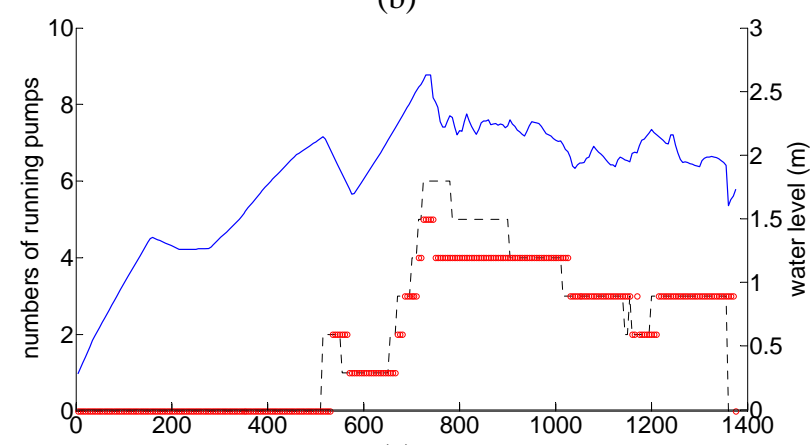

(c)

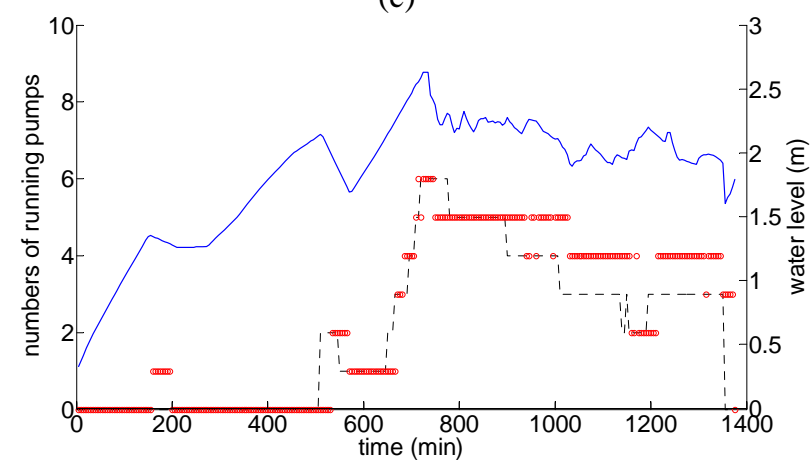

(d)

Fig. 4. The best results obtained from CFNN for lead time of (a) 5 , (b) 10 , (c) 15, and (d) $20 \mathrm{~min}$ in the testing phase.

shaded cells show in Tables 4 and 5, the ANFIS-based improved Case 1 has the best pumping operation predictions at a lead time of $5 \mathrm{~min}$ and improved Case 2, Case 3, and Case 4 produce more precise predictions at lead times of 10, 15,
Table 4. Results obtained from ANFIS in the validation phase.

\begin{tabular}{llrrrr}
\hline Validation & Criterion & \multicolumn{4}{c}{ Lead time } \\
& & $t+5$ & $t+10$ & $t+15$ & $t+20$ \\
\hline Conventional type & MAE & 0.04 & 0.10 & 0.17 & 0.21 \\
& MSE & 0.05 & 0.13 & 0.21 & 0.26 \\
\hline Improved Case 1 & MAE & 0.03 & 0.08 & 0.16 & 0.18 \\
& MSE & 0.04 & 0.10 & 0.18 & 0.26 \\
\hline Improved Case 2 & MAE & & 0.07 & 0.13 & 0.16 \\
& MSE & & 0.09 & 0.18 & 0.24 \\
\hline Improved Case 3 & MAE & & & 0.12 & 0.18 \\
& MSE & & & 0.15 & 0.26 \\
\hline Improved Case 4 & MAE & & & & 0.16 \\
& MSE & & & & 0.22 \\
\hline
\end{tabular}

Table 5. Results obtained from ANFIS for in the testing phase.

\begin{tabular}{llrrrr}
\hline Testing & Criterion & \multicolumn{4}{c}{ Lead time } \\
& & $t+5$ & $t+10$ & $t+15$ & $t+20$ \\
\hline Conventional type & MAE & 0.03 & 0.04 & 0.08 & 0.08 \\
& MSE & 0.04 & 0.05 & 0.10 & 0.11 \\
\hline Improved Case 1 & MAE & 0.02 & 0.04 & 0.07 & 0.06 \\
& MSE & 0.03 & 0.05 & 0.08 & 0.10 \\
\hline Improved Case 2 & MAE & & 0.03 & 0.05 & 0.07 \\
& MSE & & 0.05 & 0.08 & 0.11 \\
\hline Improved Case 3 & MAE & & & 0.04 & 0.06 \\
& MSE & & & 0.07 & 0.10 \\
\hline Improved Case 4 & MAE & & & & 0.05 \\
& MSE & & & & 0.09 \\
\hline
\end{tabular}

and $20 \mathrm{~min}$, respectively. The dissimilarities between CFNNbased and ANFIS-based pumping operation models are the efficiency of models and the accuracy of outputs. The number of rules built in each ANFIS model is no more than five, which is much smaller than the number of rules created in CFNN models. The results also demonstrate that the subtractive fuzzy clustering algorithm can enhance the capability of ANFIS to effectively depict the input-output patterns by using the minimum rules.

Regarding the accuracy of model predictions, the ANFIS models produce more precise predictions than CFNN models in terms of lower MAE and MSE (see Tables 3 and 5). Apparently, the ANFIS-based prediction models in this case study of pumping operations are more effective than the CFNN-based models. Figure 5 presents the comparisons between ANFIS operations and actual operations and the corresponding error patterns at different forecast lead times in testing phase. Obviously, the ANFIS-based model shows its excellent capability for pumping operation predictions up to a lead time of $20 \mathrm{~min}$, and its results outperform those obtained 

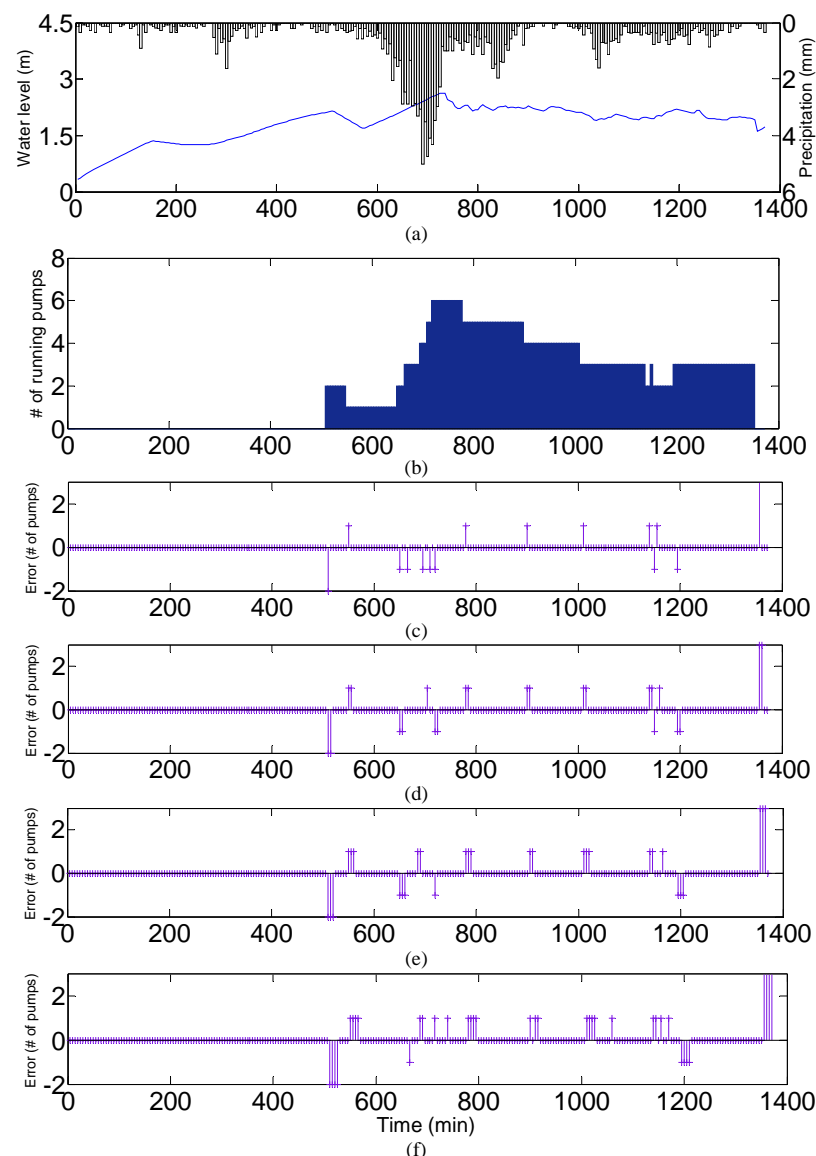

Fig. 5. The best operations obtained from ANFIS in the testing phase (a) the precipitation and water level, (b) real operations, and the corresponding error pattern for (c) 5-, (d) 10-, (e) 15-, and (f) 20-min-ahead prediction.

from CFNN model. Basically, the error computed from real records and ANFIS outputs is within one pump during high water level periods. From the error distribution shown in Fig. 6, it is evident that most of the predictions produced by ANFIS models are very precise according to the bias values. Predictions with positive bias meant overestimations, while underestimations were indicated as negative bias. Basically, results displayed in Figs. 5 and 6 demonstrate that the predictions of pumping operations from ANFIS well fit the observations without significant overestimation or underestimation existing in model outputs.

Figure 7 displays the patterns of accumulative absolute error (AAE) calculated from CFNN and ANFIS. Basically, the CFNN model produces similar patterns for forecast lead time up to 15 min with an AAE of about 40, 60, and 70 pumps for 5-, 10-, and 15-min-ahead predictions, respectively. However, the CFNN does not seem to capture the trend of pumping operations and thus results in an error of 140 pumps for 20-min-ahead predictions. Whereas, it is clear that the error patterns generated by ANFIS are very consistent and stable

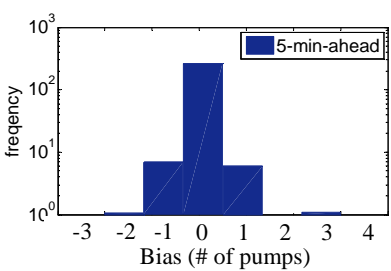

(a)

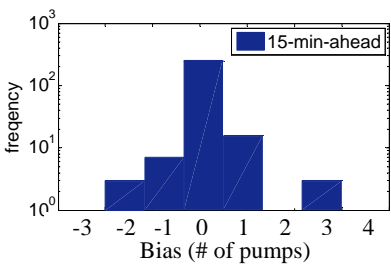

(c)

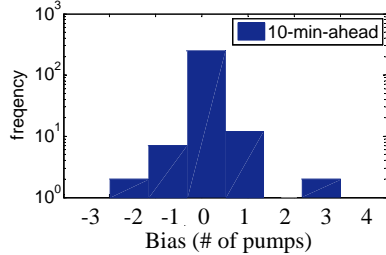

(b)

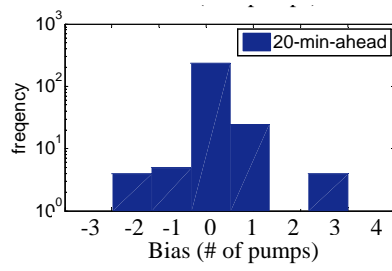

(d)
Fig. 6. The distribution of testing error calculated from real records and ANFIS outputs for lead time of (a) 5 (b) 10 (c) 15 and (d) 20 $\min$.

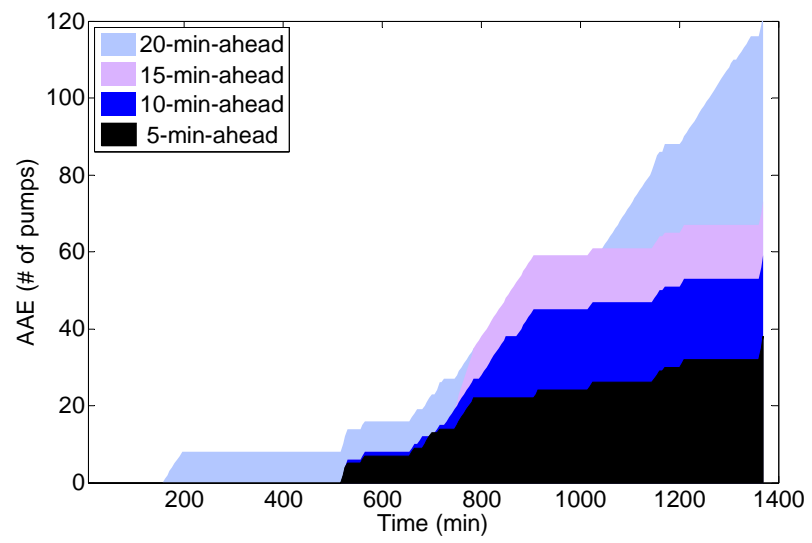

(a)

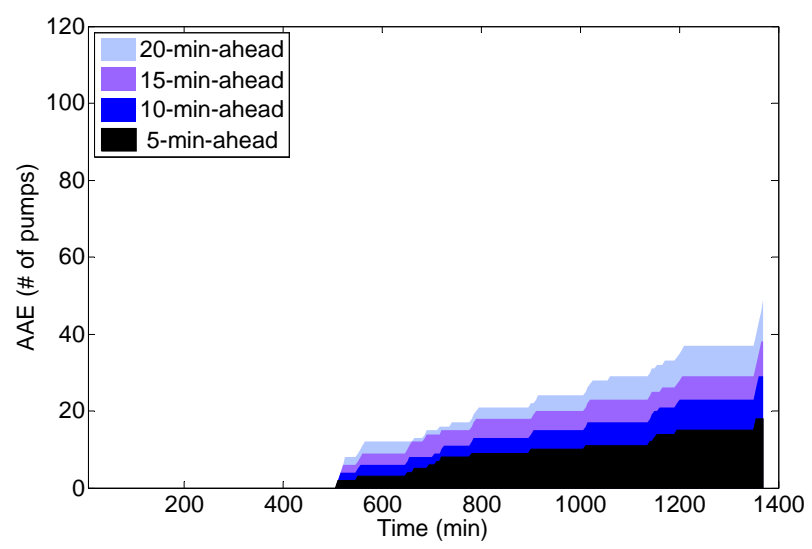

(b)

Fig. 7. The patterns of accumulative absolute error obtained from (a) CFNN and (b) ANFIS. 
with the AAE less than 20, 30, 40, and 50 pumps for lead time up to $20 \mathrm{~min}$. Table 6 gives a detailed inspection of predictions obtained from ANFIS-based pumping operation models for different forecast lead times in terms of correctness. The correctness was calculated from the number of actual running pumps and the prediction values of the model. For 5-min-ahead prediction, all correctness of model predictions for different numbers of running pumps is higher than $90 \%$ except for two sets of running pumps with correctness of $81 \%$.

To sum up, the overall correctness of ANFIS-based pumping operation models for a forecast lead time of $5 \mathrm{~min}$ is about $95 \%$, indicating the prediction is applicable to pumping operations in urban sewerage systems. The results for lead times of 10, 15, and 20 min maintain high accuracy with overall correctness of $92 \%, 90 \%$, and $87 \%$, respectively, which is much better than those of CFNN with the overall correctness decreasing from $88 \%$ to $59 \%$. Besides, when the number of running pumps is more than four sets, the precisions achieved by ANFIS models are all higher than $80 \%$, which demonstrates that the constructed model is reliable even when the water level is high. Based on the comprehensive comparison, it is convincing that the ANFIS models can be successfully applied to the operations of pumping stations in practice. Furthermore, the ANFIS-based operating model in the testing phase showed that the model maintained a consistent performance (see Tables 5 and 6). Therefore, the proposed fuzzy control system is considered to have a great capability to learn from human knowledge/experience and make suitable decisions and is able to provide consistent and reliable performance.

\section{Conclusions}

The major purpose of this study is to construct an accurate pumping operation model to effectively drain rainwater away in time and avoid the occurrence of flooding because typhoons often bring heavy rainfalls. To achieve this goal, two rule-based fuzzy neural networks, CFNN and ANFIS, are applied to predict the operation of a pumping station in an urban sewerage system in Taipei City. Both models combine the features of artificial neural network and fuzzy logic to tune the complicated conversion of human intelligence to pumping operating systems. Two input types were adopted for separately training the CFNN- and ANFIS-based forecasting models to identify the contribution of predictive water level information to pumping operations. The reliability and predictability of both models fed with predictive water levels were also explored based on various inputs for forecast lead time up to $20 \mathrm{~min}$.

A comprehensive comparison indicates that the constructed ANFIS model provides better performance in predicting the operations of a pumping station than the CFNN model. The similarity percentage, in terms of correctness,
Table 6. Performance of ANFIS-based pumping operation in testing phase.

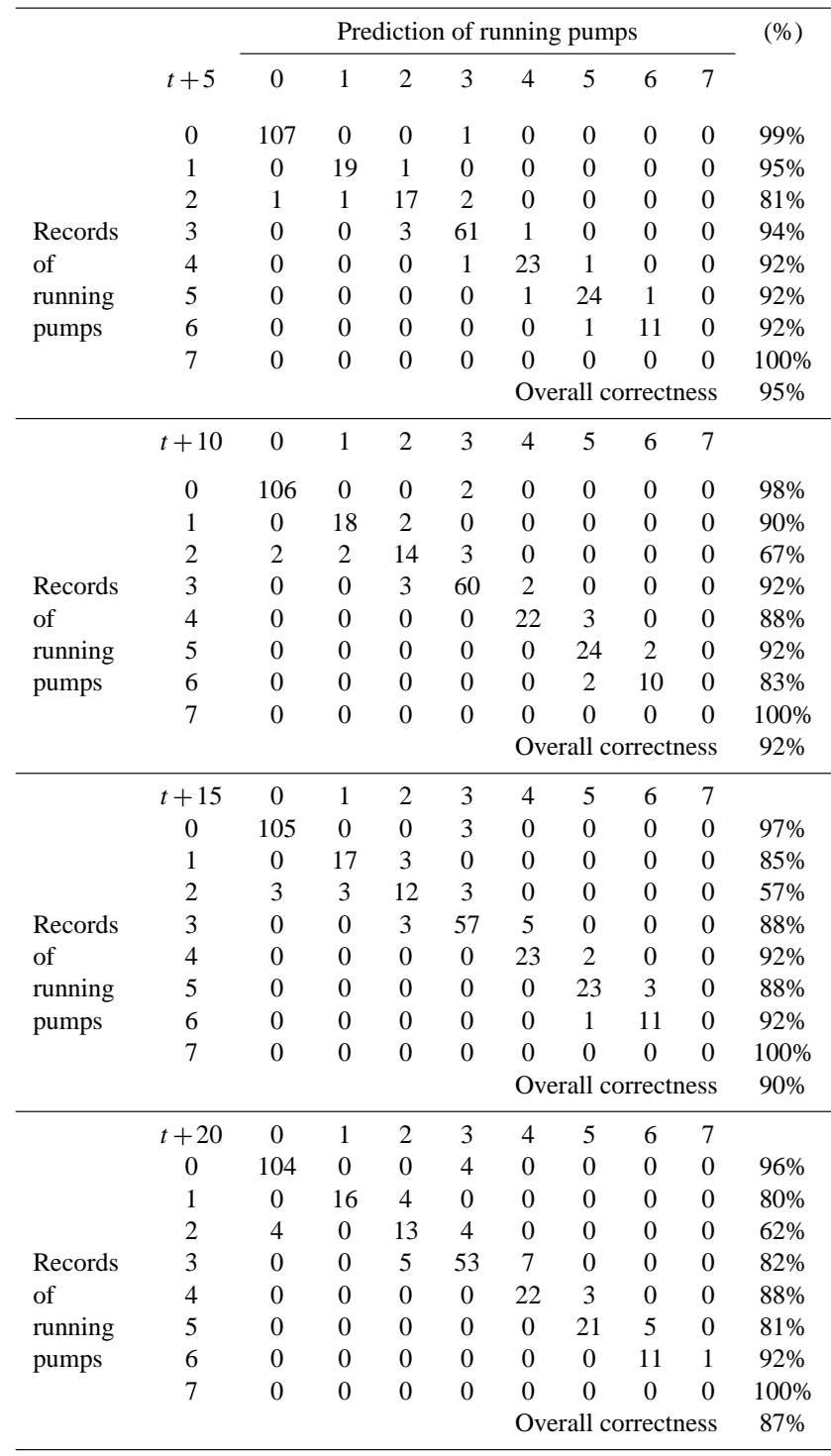

of running pumps between the ANFIS predictions and historical records is higher than $87 \%$ for 20 -min-ahead prediction, which is much better than $59 \%$ obtained from the CFNN model. ANFIS not only outperforms CFNN in model accuracy but also in model efficiency. CFNN has to build at least fifteen and up to twenty-eight rules to describe the relation between input variables and pumping operations for different scenarios, whereas the number of rules required for $\mathrm{AN}$ FIS is less than five, which is adequate to simulate a complex nonlinear system. As far as the same forecast lead time is concerned, the best performance is always achieved in the model fed with the latest predictive water level information. This also implies that small bias existing in the predictive 
water levels may result in a larger error if the pumping operation model is designed for longer forecast lead time. Issues regarding such analysis will be an interesting topic for future study. Overall, the study gives a preliminary investigation on the applicability of rule-based fuzzy neural networks to urban sewerage systems and the contribution of predictive information to the operations of pumping stations. It is clear that the ANFIS model can efficiently describe the relation between input patterns and pumping operations via minimum rules and provide precise predictions of running pumps.

Acknowledgements. This research was sponsored by the Water Resource Agency, Ministry of Economic Affairs, Taiwan, Republic of China. The Taipei City Government provided the sewerage gauge data for analysis objectives in this research.

Edited by: A. Montanari

\section{References}

Abrahart, R. J. and See, L.: Multi-model data fusion for river flow forecasting: an evaluation of six alternative methods based on two contrasting catchments, Hydrol. Earth Syst. Sci., 6, 655-670, doi:10.5194/hess-6-655-2002, 2002.

Brath, A., Montanari, A., and Toth, E.: Neural networks and nonparametric methods for improving real-time flood forecasting through conceptual hydrological models, Hydrol. Earth Syst. Sci., 6, 627-639, doi:10.5194/hess-6-627-2002, 2002.

Chang, F. J. and Chang, Y. T.: Adaptive neuro-fuzzy inference system for prediction of water level in reservoir, Adv. Water Res., 29(1), 1-10, 2006.

Chang, F. J. and Chen, Y. C.: A counterpropagation fuzzy-neural network modeling approach to real time streamflow prediction, J. Hydrol., 245(1-4), 153-164, 2001.

Chang, F. J., Chang, K. Y., and Chang, L. C.: Counterpropagation fuzzy-neural network for city flood control system, J. Hydrol., 358(1-2), 24-34, 2008.

Chang, L. C. and Chang, F. J.: Intelligent control for modelling of real-time reservoir operation, Hydrol. Process., 15(9), 16211634, 2001.

Chang, Y. T., Chang, L. C., and Chang, F. J.: Intelligent control for modeling of real-time reservoir operation, part II: artificial neural network with operating rule curves, Hydrol. Process., 19(7), 1431-1444, 2005.

Chaves, P. and Kojiri, T.: Deriving reservoir operational strategies considering water quantity and quality objectives by stochastic fuzzy neural networks, Adv. Water Res., 30(5), 1329-1341, 2007.

Chiang, Y. M., Chang, L. C., and Chang, F. J.: Comparison of staticfeedforward and dynamic-feedback neural networks for rainfallrunoff modeling, J. Hydrol., 290(3-4), 297-311, 2004.

Chiang, Y. M., Chang, F. J., Jou, B. J. D., and Lin, P. F.: Dynamic ANN for precipitation estimation and forecasting from radar observations, J. Hydrol., 334(1-2), 250-261, 2007.

Chiang, Y. M., Chang, L. C., Tsai, M.-J., Wang, Y.-F., and Chang, F.-J.: Dynamic neural networks for real-time water level predictions of sewerage systems-covering gauged and ungauged sites,
Hydrol. Earth Syst. Sci., 14, 1309-1319, doi:10.5194/hess-141309-2010, 2010.

Coulibaly, P. and Evora, N. D.: Comparison of neural network methods for infilling missing daily weather records, J. Hydrol., 341(1-2), 27-41, 2007.

Dawson, C. W., Harpham, C., Wilby, R. L., and Chen, Y.: Evaluation of artificial neural network techniques for flow forecasting in the River Yangtze, China, Hydrol. Earth Syst. Sci., 6, 619-626, doi:10.5194/hess-6-619-2002, 2002.

Firat, M.: Comparison of Artificial Intelligence Techniques for river flow forecasting, Hydrol. Earth Syst. Sci., 12, 123-139, doi:10.5194/hess-12-123-2008, 2008.

Hecht-Nielsen, R.: Counterpropagation Networks, Appl. Optics, 26(23), 4979-4984, 1987.

Hsu, N. S. and Wei, C. C.: A multipurpose reservoir real-time operation model for flood control during typhoon invasion, J. Hydrol., 336(3-4), 282-293, 2007.

Jang, J. S. R.: ANFIS - Adaptive-network-based fuzzy inference system, IEEE Transactions on Systems Man and Cybernetics, 23(3), 665-685, 1993.

Mehta, R. and Jain, S. K.: Optimal Operation of a Multi-Purpose Reservoir Using Neuro-Fuzzy Technique, Water Resour. Manage., 23(3), 509-529, 2009.

Nayak, P. C., Sudheer, K. P., Rangan, D. M., and Ramasastri, K. S.: A neuro-fuzzy computing technique for modeling hydrological time series, J. Hydrol., 291(1-2), 52-66, 2004.

Nie, J. H. and Linkens, D. A.: Fast Self-Learning Multivariable Fuzzy Controllers Constructed from a Modified CPN Network, Int. J. Control, 60(3), 369-393, 1994.

Pinthong, P., Gupta, A. D., Babel, M. S., and Weesakul, S.: Improved Reservoir Operation Using Hybrid Genetic Algorithm and Neurofuzzy Computing, Water Resour. Manage., 23(4), 697-720, 2009.

Rao, S. V. N., Kumar, S., Shekhar, S., Sinha, S. K., and Manju, S.: Optimal pumping from skimming wells from the Yamuna River flood plain in north India, Hydrogeol. J., 15(6), 1157-1167, 2007.

Shrestha, R. R. and Nestmann, F.: Physically Based and DataDriven Models and Propagation of Input Uncertainties in River Flood Prediction, J. Hydrol. Eng., 14(12), 1309-1319, 2009.

Sudheer, K. P., Chaubey, I., and Garg, V.: Lake water quality assessment from Landsat thematic mapper data using neural network: An approach to optimal band combination selection, J. Am. Water Resour. Assoc., 42(6), 1683-1695, 2006.

Toth, E.: Classification of hydro-meteorological conditions and multiple artificial neural networks for streamflow forecasting, Hydrol. Earth Syst. Sci., 13, 1555-1566, doi:10.5194/hess-131555-2009, 2009.

Tyagi, P., Chandramouli, V., Lingireddy, S., and Buddhi, D.: Relative performance of artificial neural networks and regression models in predicting missing water quality data, Environ. Eng. Sci., 25(5), 657-668, 2008.

Wang, W. C., Chau, K. W., Cheng, C. T., and Qiu, L.: A comparison of performance of several artificial intelligence methods for forecasting monthly discharge time series, J. Hydrol., 374(3-4), 294-306, 2009.

Yarar, A., Onucyildiz, M., and Copty, N. K.: Modelling level change in lakes using neuro-fuzzy and artificial neural networks, J. Hydrol., 365(3-4), 329-334, 2009. 